\title{
Structure-Based Evolutionary Programming Design of Broadband Wire Antennas
}

\author{
Giovanni Andrea Casula, Giuseppe Mazzarella, and Giorgio Montisci \\ Dipartimento di Ingegneria Elettrica ed Elettronica, Università di Cagliari, Piazza D’Armi, 09123 Cagliari, Italy \\ Correspondence should be addressed to Giuseppe Mazzarella; mazzarella@diee.unica.it
}

Received 3 October 2012; Revised 10 December 2012; Accepted 21 December 2012

Academic Editor: Renato Cicchetti

Copyright (C) 2013 Giovanni Andrea Casula et al. This is an open access article distributed under the Creative Commons Attribution License, which permits unrestricted use, distribution, and reproduction in any medium, provided the original work is properly cited.

\begin{abstract}
A design technique for wire antennas, based on the Structure-Based Evolutionary Programming, is used to design a broadband antenna with an end-fire radiation pattern and a very simple geometry, operating in the 3-16 GHz frequency band, namely, from the $\mathrm{S}$ band to the Ku band. The antenna has been analyzed with NEC-2 during the evolutionary process, looking for high gain, good input match, and robustness with respect to realization tolerances. The outcome of our design procedure shows a very good performance.
\end{abstract}

\section{Introduction}

Antenna design has been a matter of intuition and brute-force computation from the beginning (see e.g., $[1,2])$. In the past literature, this task has been faced at different levels, from simple formulas [3] to sophisticated synthesis techniques [4$11]$, heuristic models $[12,13]$, or one of the different random optimization procedures proposed so far [14-18].

The traditional approach to the design of wire antennas starts by choosing a well-defined structure, whose parameters need to be suitably optimized. This requires, as a prerequisite, the choice of an antenna model which has proven able to comply with the design specifications. Successful proposals of broadband antennas are self-similar, so for those antennas the chosen model must be a self-similar one, either continuous, like a conical antenna, or discrete. The latter can be implemented either as an array [12] or as a prefractal antenna [19]. Of course, the final antenna could not fulfill exactly the model [13], but also such differences must be chosen externally. As a matter of fact, the choice of the model heavily constraints the whole design process. As a consequence, a significant skilled human interaction is required in the initial choice of the structure. The model could also be fine-tuned as the design proceeds, but this requires a skilled human interaction, too. The traditional approach is quite expensive, and therefore design techniques without human interaction are of interest, as long as they provide equal, or better, results. This can be achieved only when no initial structure is assumed, since this choice (which in a fully automated procedure cannot be further modified) can constraint too strongly the final solution.

Up to now, such a general tool has been sought for among random search procedures. Some of them are inspired by natural processes, either looking for an effective cooperative scheme, like Particle Swarm Optimization (PSO) [20], or aiming at exploring very different solution sets, like Genetic Algorithms (GA) [21-23]. As a matter of fact, the latter could be a good candidate for a valuable general tool, being inspired by Darwinian natural selection, since the variety of all living forms is known to everyone. However, despite of their strong premises, GA cannot fulfill this purpose since they still assume a completely defined antenna structure, and only an handful of parameters remains to be optimized. Borrowing the biological language, we can say that GA works at the nucleotide (i.e., bit) level and this strongly limits its effectiveness as a design tool.

On the other hand, the full power of evolution-inspired methods in the antenna design has been highlighted only when Genetic Programming (GP) or, more precisely, Structure-based Evolutionary Design (SED) [24] approaches have been proposed for simple wire antennas $[25,26]$, arrays [24], and FSS [27]. Evolutionary programming does not 
require any antenna model, neither asks for a structure locked from the beginning. Instead, it considers a (virtually) infinite solution space, defined only by very loose constraints. If we require a wire antenna, $\mathrm{SED}$ is able to look for the final design among all possible wire antennas. As a matter of fact, SED can be used to automatically, and effectively, search, in this very huge solution space, for novel antenna configurations, which can be significantly more performant than antennas developed using standard techniques.

The strength of the SED resides in its description of each element of the solution space as the set of instructions needed to realize it. This description can be translated in a highly effective tree description [25] for computer implementation and allows the standard genetic operators (crossover, mutation) to reach an unparallel power. As a matter of fact, SED works at the organ level, so that crossover is the exchange of whole working parts of the individuals, while mutation, working on a subtree root, affects the whole sub-tree (as happens in the true natural selection). SED requires also a suitable fitness function, tailored to the problem at hand, and a time-effective analysis procedure.

To assess SED as a viable tool for robust design of broadband antennas, we consider here the design of a wideband wire antenna with an end-fire radiation pattern and a very simple geometry, operating in a range from $\mathrm{S}$ to $\mathrm{Ku}$ frequency bands, namely, from $3 \mathrm{GHz}$ to $16 \mathrm{GHz}$, and reasonably matched at the input port. Apart from these simple "constraints," SED does not assume any other a priori information on the antenna structure. Rather, SED builds up the structure of the individual antennas as the procedure evolves. Therefore, SED solution space has the power of the continuum and allows exploring, and evaluating, general wire antenna configurations.

The solution space, namely, the set of admissible solutions in which the procedure looks for the optimum, is composed, in our case, of every broadband antenna with no limit on the number of wire segments, nor on the size or orientation, represented as real numbers. On the other hand, GA works on a given antenna model, so that its solution space is a discrete one and therefore is a very small subset of the SED solution space.

The most common broadband wire antennas are the log-periodic dipole arrays (LPDAs) [28-30], used in a wide range of applications due to their very wide bandwidth and their relatively narrow-beam characteristics. However, a wire LPDA operating in the bandwidth of the antenna proposed in this paper (namely, C, X, and Ku frequency bands) cannot be realized, because the corresponding dipoles would be too short at these frequencies. On the other hand, the broadband antenna designed here with SED can be easily scaled in order to work also at lower frequencies, keeping the same performances both in terms of input matching and of gain.

In order to compute the fitness, an analysis of each wire antenna generated by SED is needed. This has been performed using NEC-2 [31], a well-known, time-effective and well-assessed Method of Moments code. This software has been successfully used to model a wide range of wire antennas, with high accuracy (see, e.g., $[32,33]$ ), and is now considered as one of the reference e.m. software (see, e.g.,

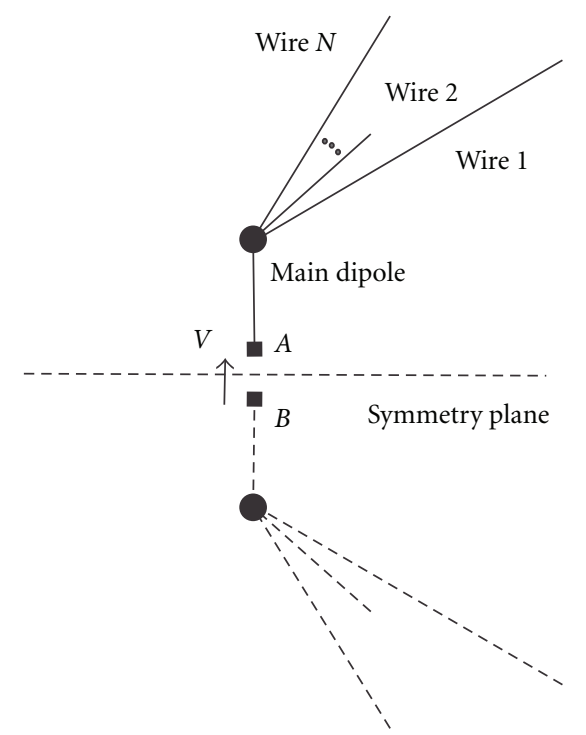

Figure 1: Wire antenna geometry.

$[26,34])$. For this reason, it has been used here. However, sometimes the SWR data of NEC-2 could have a reduced accuracy, therefore the final output of the design procedure has been validated, by testing it with HFSS [35], a commercial FEM code, since it has been shown that the results of this software are in very good agreement with experiment (see, e.g., [36]). As a matter of fact, although NEC is faster by orders of magnitude, NEC and HFSS results are in very good agreement, thus assessing our choice for the fitness evaluation.

\section{Antenna Design and Fitness Function}

The initial structure of each SED individual is depicted in Figure 1.

Each individual of the population (antenna) is composed by a principal vertical wire (the main dipole in Figure 1), connected to the feeding port on its bottom side, and by a number $N$ (chosen by SED) of wires connected to the upper side of the main dipole with an arbitrary length and orientation in space. At the remote end of each of the $N$ wires, we connect zero, one, or more further wires, still with arbitrary length and orientation, and so on, in an iterative manner. The structure is finally mirrored with respect to the horizontal plane, as indicated in Figure 1.

Each individual is built up using only four operations:

(a) add a wire according to the present directions and length;

(b) transform the end of the last added wire in a branching point;

(c) modify the present directions and length;

(d) stretch (or shrink) the last added wire.

In the first step of the evolutionary design, $N$ individuals are randomly built. Then, an iterative procedure starts, where 
the fitness of each individual is evaluated, and the next generation of the population is built assigning a larger probability of breeding to the individuals with the highest fitness. The iterative procedure ends when suitable stopping rules are met (i.e., when the individual antenna fulfills, within a predetermined tolerance, the specified requirements).

After each antenna has been generated, its geometrical coherency is verified, and incoherent antennas (e.g., an antenna with two elements too close, or even intersecting) are discarded. Then it is analysed by NEC- 2 and its fitness is computed. The SED approach has been implemented in Java, while the analysis of each individual has been implemented in $\mathrm{C}++$ (using the freeware source code Nec2cpp) and checked using the freeware tool 4 nec2 [31].

The performance of each individual (antenna) of the population is evaluated by a proper fitness function, which is strongly dependent on the problem at hand, namely, by the electromagnetic behavior of the designed antenna, and must measure how closely the actual antenna meets the design specifications.

In the specific case of the broadband wire antenna of this paper, the fitness function has been selected in order to lead the evolution process toward a structure with a good input match in a frequency range as wide as possible (within S, C, $\mathrm{X}$, and $\mathrm{Ku}$ bands), while keeping the highest end-fire gain and a reduced size.

Since improving one parameter usually results in worsening the other ones, the design technique has to handle a complicate trade-off between the conflicting objectives. The fitness structure is therefore a critical point in the design procedure, since only an appropriate choice can lead the design process to performing results, while largely reducing the computation time.

The chosen fitness has been built from the desired antenna performances [24] as

$$
\begin{aligned}
\text { Fitness }= & {\left[(1-\overline{\mathrm{SWR}}) \cdot \alpha_{\mathrm{SWR}}+\frac{G_{\mathrm{MAX}}}{\bar{G}} \cdot \alpha_{\mathrm{GAIN}}\right] } \\
& \cdot\left(1+K_{\mathrm{SIZE}} \cdot \frac{D_{\mathrm{MAX}}}{D_{\mathrm{ANT}}}\right),
\end{aligned}
$$

where $\alpha_{\text {SWR }}$ and $\alpha_{\text {GAIN }}$ are suitable weights (whose values depend also on the input impedance of the actual antenna), $\overline{S W R}$, and $\bar{G}$ are, respectively, the mean values of the SWR and gain over the bandwidth of interest, $D_{\text {ANT }}$ represents the actual antenna size, and $D_{\mathrm{MAX}}$ is the maximum allowed size for the antenna. Finally, $K_{\text {SIZE }}$ is an appropriate weight which takes into account the requirement of a small size of the antenna. The values for the fitness weights have been obtained after a suitable local tuning, following an approach similar to the one described in detail in [24].

The weight $\alpha_{\text {GAIN }}$ in the fitness function (1) has the following expression:

$$
\begin{aligned}
\alpha_{\mathrm{GAIN}}= & \left(1+\alpha_{\text {Back }} * G_{\text {Back }}\right) \cdot\left(1+\alpha_{\text {Rear }} * G_{\text {Rear }}\right) \\
& \cdot\left(1+\alpha_{\text {Front }} * G_{\text {Front }}\right),
\end{aligned}
$$

where $G_{\text {Back }}$ is the gain computed in the back direction $(\theta=$ $\left.90^{\circ}, \varphi=180^{\circ}\right), G_{\text {Front }}$ is the average gain computed in the front region $\left(|\theta|>90^{\circ}+2 \Delta 9,0^{\circ}+2 \Delta \varphi<\varphi<90^{\circ}\right.$, where $\Delta \vartheta$ and $\Delta \varphi$ indicate the main lobe amplitude), and $G_{\text {Rear }}$ is the average gain computed in the rear region $\left(0^{\circ} \leq|\mathcal{Y}| \leq\right.$ $180^{\circ}, 90^{\circ} \leq|\varphi| \leq 180^{\circ}$ ). The weights $\alpha_{\text {Back }}, \alpha_{\text {Front }}$ and $\alpha_{\text {Rear }}$ are chosen through a local tuning in order to get the maximum gain in the end-fire direction and an acceptable radiation pattern in the rest of the space. In the performed evolutionary process these parameters have the following values: $\alpha_{\text {Back }}=$ $0.12, \alpha_{\text {Front }}=0.17$ and, $\alpha_{\text {Rear }}=0.06$.

The weight $\alpha_{\text {SWR }}$ in the fitness function (1) is expressed using suitable parameters strictly related to the antenna input impedance, which are individually tuned. The resulting expression for $\alpha_{\text {SWR }}$ is

$$
\begin{aligned}
\alpha_{\mathrm{SWR}}= & \left(1+\alpha_{\mathrm{IN}}\right) \cdot\left(1+\alpha_{X}\left|X_{\mathrm{IN}}^{A}\right|\right) \cdot\left(1+\alpha_{\mathrm{Q}} \frac{R_{\mathrm{IN}}^{A}-\left|X_{\mathrm{IN}}^{A}\right|}{R_{\mathrm{IN}}^{A}}\right) \\
& \cdot\left(1+\alpha_{\mathrm{Var} R} \cdot \sigma_{R}^{2}\right) \cdot\left(1+\alpha_{\mathrm{Var} X} \cdot \sigma_{X}^{2}\right),
\end{aligned}
$$

where

(i) $\alpha_{\mathrm{IN}}=50$ if $\left|X_{\mathrm{IN}}^{A}\right|>R_{\mathrm{IN}}^{A}$, and $\alpha_{\mathrm{IN}}=0$ otherwise (weight introduced in order to boost up structures with $\left.R_{\mathrm{IN}}^{A}>\left|X_{\mathrm{IN}}^{A}\right|\right)$;

(ii) $\alpha_{X}=0.12$ (weight related to $\left|X_{\mathrm{IN}}^{A}\right|$, introduced in order to force the evolution process to structures with an $\left|X_{\text {IN }}^{A}\right|$ as small as possible);

(iii) $\alpha_{\mathrm{Q}}=0.2$ (weight related to $R_{\mathrm{IN}}^{A}-\left|X_{\mathrm{IN}}^{A}\right|$, and introduced to advantage structures with a low $Q$ factor);

(iv) $\alpha_{\operatorname{Var} R}=\alpha_{\operatorname{Var} X}=0.03$ (weight related to the normalized mean square variation of $R_{\mathrm{IN}}^{A}$ and $X_{\mathrm{IN}}^{A}$ in the antenna required bandwidth, and introduced to advantage structures with a regular impedance behaviour);

and $R_{\mathrm{IN}}^{A}$ and $X_{\mathrm{IN}}^{A}$ are, respectively, the real part and the imaginary part of the antenna input impedance, while $\sigma_{R}^{2}$ and $\sigma_{X}^{2}$ are the normalized mean square variation of $R_{\mathrm{IN}}^{A}$ and of $X_{\mathrm{IN}}^{A}$ in the antenna required bandwidth. The two weights $\alpha_{\mathrm{IN}}$ and $\alpha_{\mathrm{Q}}$ are both connected to the $\mathrm{Q}$ factor of the antenna. However, $\alpha_{\mathrm{IN}}$ gives a significant penalization to antennas with a large imaginary part of the input impedance, but it has a step-like behavior. Therefore, in order to get a further, smooth penalization to antennas with a large $Q$, we have added also the term with $\alpha_{\mathrm{Q}}$. We have observed that a combination of the two terms is more effective than either one separately.

The inclusion of ohmic losses into the gain computation, as well as the requirement of a good input match over all the required bandwidth, prevents from selecting superdirective solutions.

On the other hand, the requirement of a robust solution is not taken into account in the fitness, since we have found a different approach more efficient. The individuals associated with the highest fitness values, or very close to the 


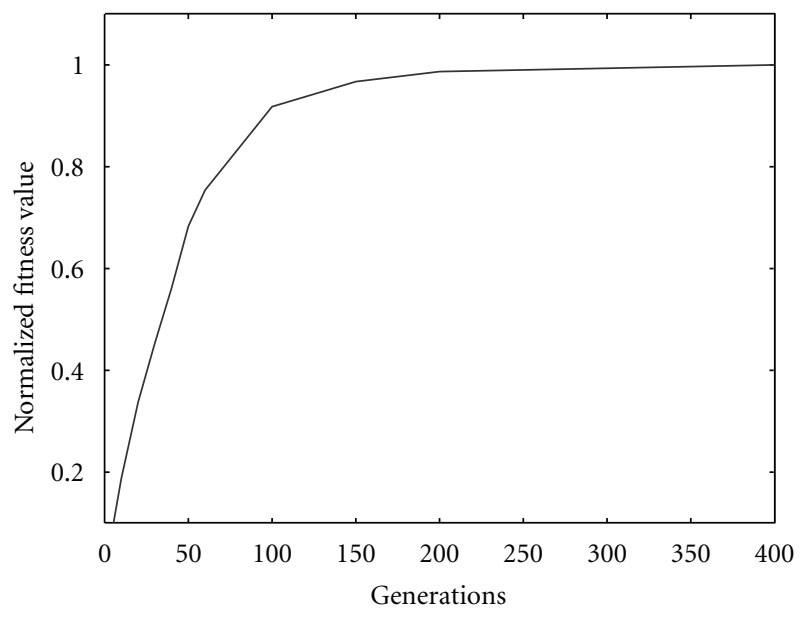

Figure 2: Plot of convergence of the designed antenna shown in Figure 3.

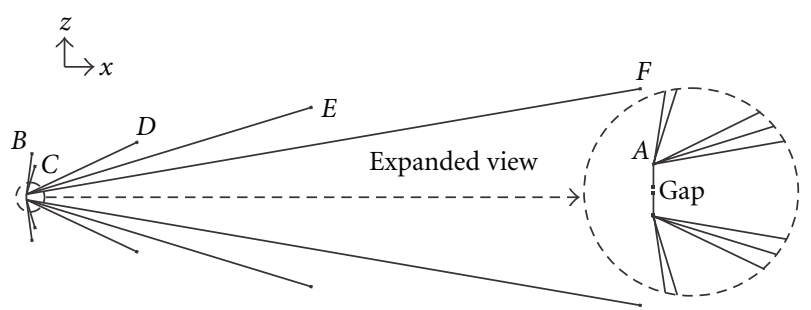

FIGURE 3: Geometry of the designed wire antenna.

best fitness value obtained so far, are perturbed (assigning random relocations to the elements) and analysed to assess their robustness with respect to random modifications of the structure. This random relocation allows to get robust structures with respect to both constructive errors and bad weather conditions (e.g., movements due to wind effect).

\section{Results}

In order to test the procedure, we have designed a broadband wire antenna, with equal wire diameter $(0.665 \mathrm{~mm})$ and conductivity $\left(\sigma=4 * 10^{6} \mathrm{~S} / \mathrm{m}\right)$. We have required that the antenna has an input impedance of $200 \Omega$ in the whole bandwidth, which is a typical characteristic impedance of bifilar lines [37]. The use of a bifilar line as a feeding network avoids the need of a balun to connect the balanced wire antenna to an unbalanced input, as provided for example by standard coaxial cables, having a typical input impedance of $50 \Omega$. On the other hand, the designed antenna can also be connected to a standard coaxial line using a commercial balun with an impedance transformation ratio of $4: 1$. In this case, since a balun with such large bandwidth $(3-16 \mathrm{GHz})$ cannot be obtained, the operating bandwidth must be divided into a number of subbands, and an appropriate balun must be used in each subband.

The antenna has been designed using a population size of 1000 individuals, with a crossover rate set to $60 \%$, and a

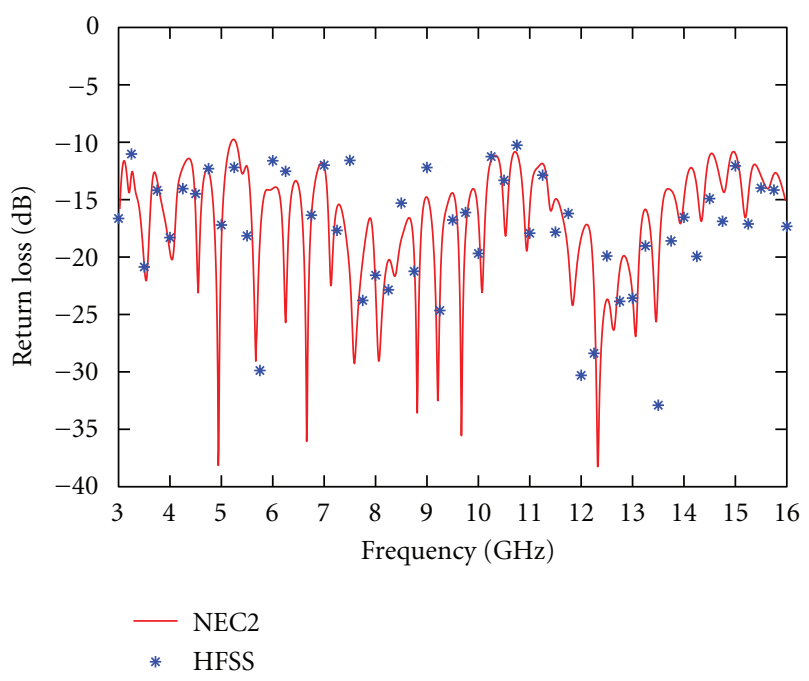

FIGURE 4: Frequency response of the designed antenna.

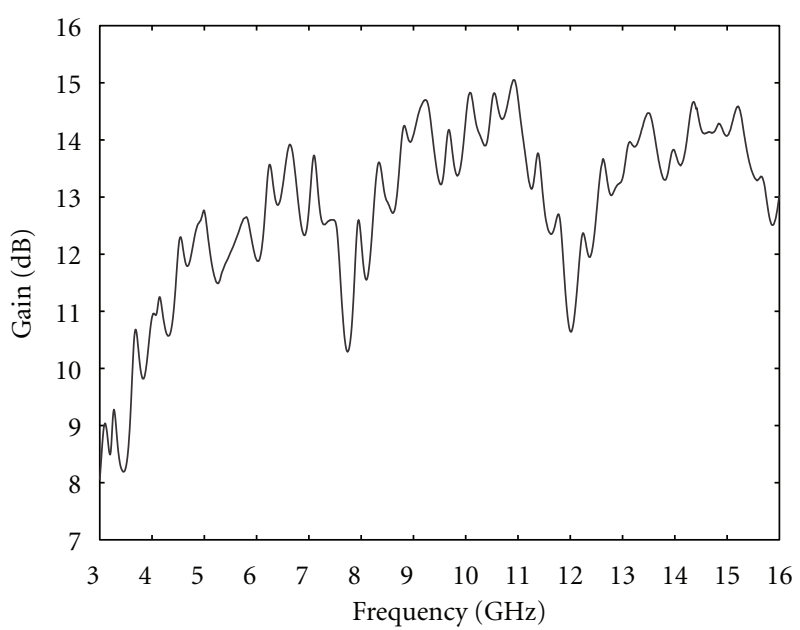

FIGURE 5: Simulated (NEC-2) Gain of the designed antenna.

mutation rate set to $40 \%$. Its convergence plot is shown in Figure 2, and it appears that 250 generations are enough to reach convergence.

The best individual of the evolutionary process, obtained after several runs (e.g., a few tens) of the code, is shown in Figure 3, and the cartesian coordinates of each wire are reported in Table 1. This antenna is very easy to realize, consisting in only 12 metallic wires, and can be produced with a very low cost by the same technology used for Yagi and LPDA arrays.

In Figure 4 we show the input frequency response of the designed antenna using both NEC-2 and HFSS. It is clear that the antenna bandwidth $(\mathrm{S} 11<-10 \mathrm{~dB})$ extends from $3 \mathrm{GHz}$ up to well beyond $16 \mathrm{GHz}$. It is also clear that both NEC- 2 and HFSS give essentially comparable results. This is also true for the radiation pattern. So, the use of NEC-2 in the design is fully assessed, and we will show only the NEC-2 results in the following. 

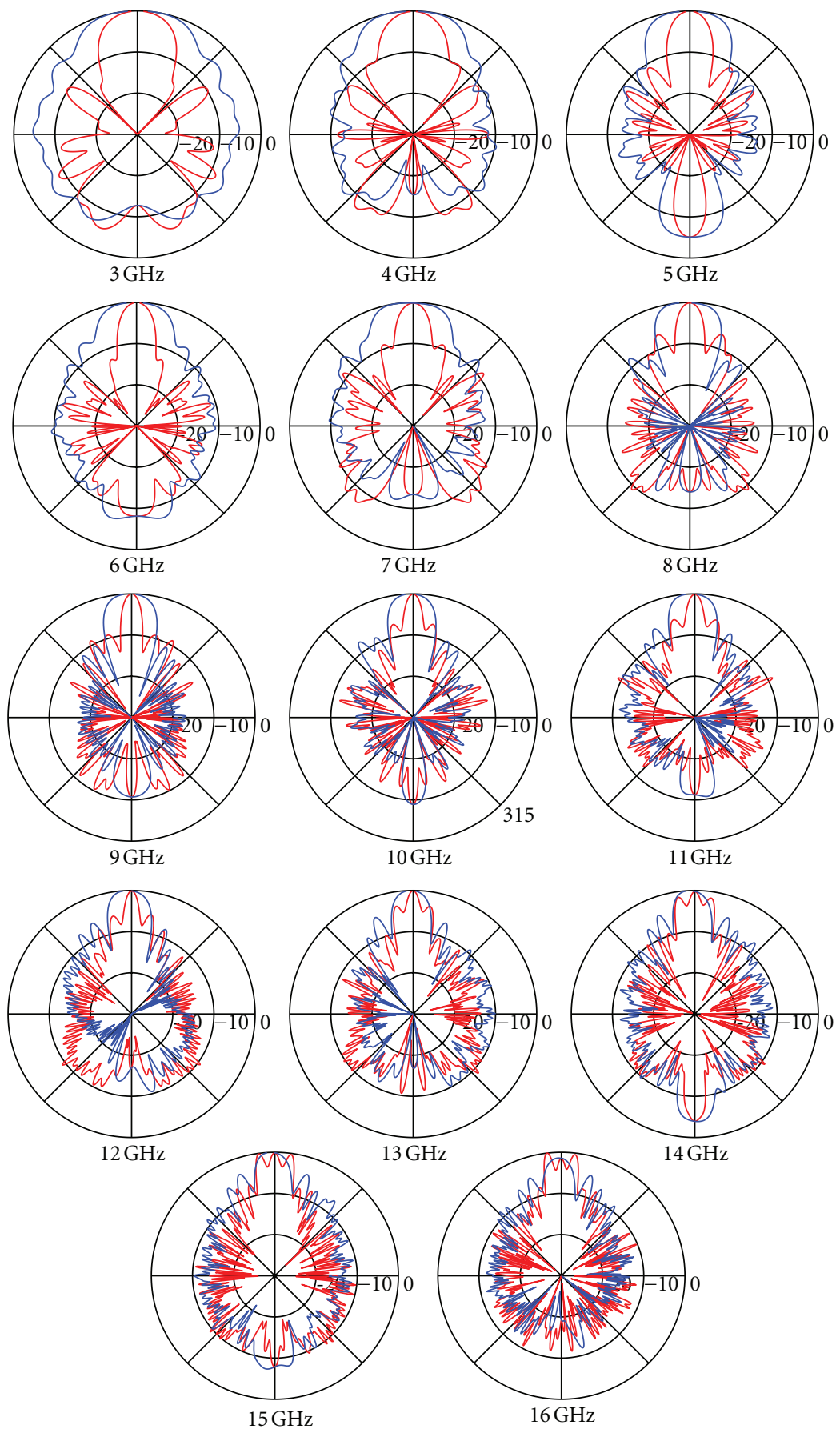

FIGURE 6: Simulated (NEC-2) normalized Far-Field pattern of the designed antenna. Blue line: E-Plane; red line: H-Plane.

In Figure 5 the end-fire Gain is reported. In the bandwidth $3-16 \mathrm{GHz}$, the mean Gain of the antenna is equal to $12.7 \mathrm{~dB}$ and the mean $\mathrm{F} / \mathrm{B}$ ratio is about $11.6 \mathrm{~dB}$.

In Table 2 the antenna gain, front-to-back ratio, and efficiency in the operating bandwidth are shown.

It is worth noting that the inclusion of ohmic losses into the gain computation is very important, since this prevents from selecting superdirective antennas during the evolution. As a matter of fact, the efficiency of the designed antenna is very good (greater than $97 \%$, and with a mean value of $98.05 \%$ ), despite of the relatively small electrical conductivity of the metal $\left(\sigma=4 * 10^{6} \mathrm{~S} / \mathrm{m}\right)$. Besides, while the maximum directivity is almost constant with respect to $\sigma$, the efficiency rapidly decreases [38]. It is therefore required to take into account in SED the actual conductivity of the antenna material, in order to discard individuals with low efficiency, which can result in unusable antennas.

Finally, the NEC-2 Far-Field-pattern in the operating frequency bandwidth is plotted in Figure 6. For each frequency, the E-Plane and the H-Plane are shown. The reported 


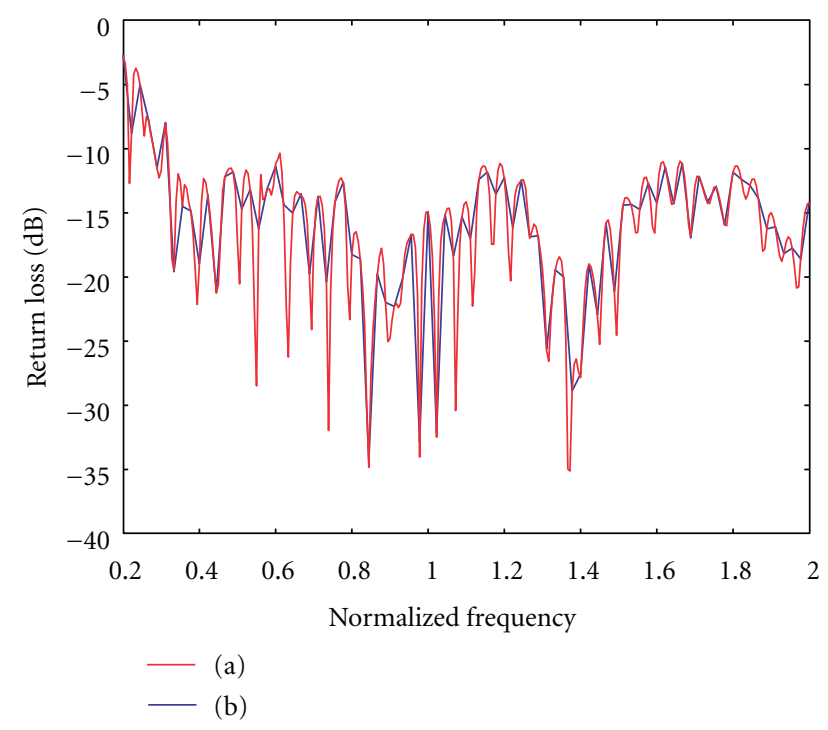

FIGURE 7: Simulated (NEC-2) Return Loss of the designed antenna at the center frequency of $8 \mathrm{GHz}$ (a) and of the designed antenna scaled at the center frequency of $2 \mathrm{GHz}(\mathrm{b})$.

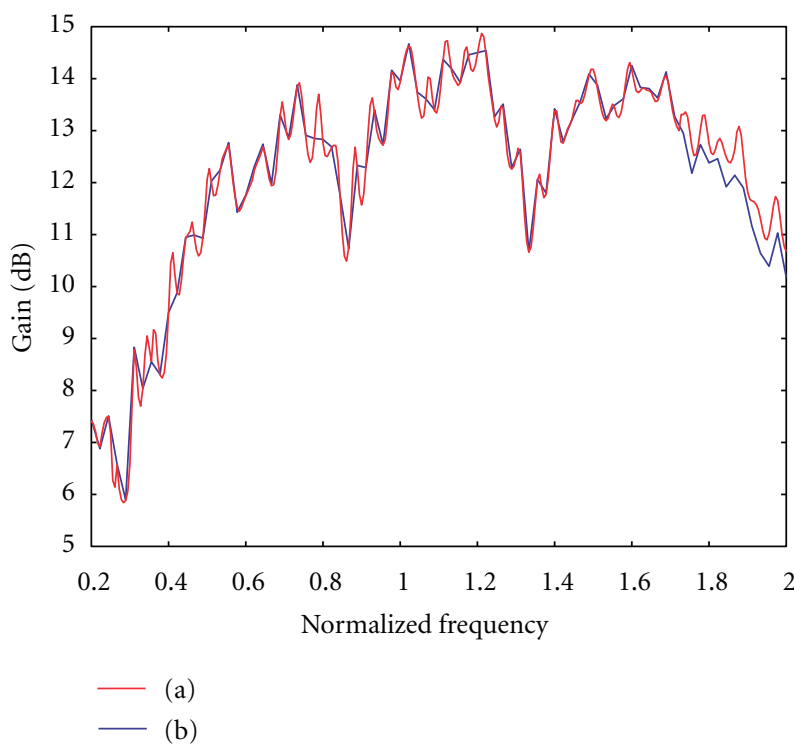

FIGURE 8: Simulated (NEC-2) Gain of the designed antenna at the center frequency of $8 \mathrm{GHz}$ (a) and of the designed antenna scaled at the center frequency of $2 \mathrm{GHz}(\mathrm{b})$.

radiation patterns confirm that the useful bandwidth of the designed antenna is $3-16 \mathrm{GHz}$, where the input matching is very good and the far-field is essentially end fire, with a good Gain and $\mathrm{F} / \mathrm{B}$ ratio.

In order to evaluate the performance improvement of the broadband antenna proposed in this paper over standard solutions, we can compare it with wire log-periodic dipole arrays (LPDAs), the most popular broadband wire antennas [28-30]. However, a wire LPDA in the $C$ frequency band (and beyond) cannot be realized because the dipole lengths would be too small with increasing frequency. On the other hand,
TABLE 1: Cartesian coordinates of the ends of the wires for the antenna in Figure 2.

\begin{tabular}{lccc}
\hline & $X(\mathrm{~mm})$ & $Y(\mathrm{~mm})$ & $Z(\mathrm{~mm})$ \\
\hline A & 0 & 0 & 2.00 \\
B & 1.97 & 2.38 & 30.25 \\
C & 2.07 & 3.35 & 20.82 \\
D & 60.25 & 5.85 & 39.50 \\
E & 156.25 & 5.85 & 68.00 \\
F & 340.00 & 5.85 & 86.25 \\
\hline
\end{tabular}

TABLE 2: Radiation parameters for the antenna in Figure 2.

\begin{tabular}{lccc}
\hline Freq $(\mathrm{GHz})$ & Gain $(\mathrm{dB})$ & $F / B(\mathrm{~dB})$ & Efficiency $(\%)$ \\
\hline 3 & 7.38 & 12.69 & 99.05 \\
4 & 10.04 & 15.33 & 98.53 \\
5 & 12.98 & 7.25 & 97.06 \\
6 & 12.60 & 8.07 & 97.84 \\
7 & 10.30 & 13.19 & 98.11 \\
8 & 12.40 & 13.87 & 98.15 \\
9 & 14.89 & 10.70 & 98.48 \\
10 & 14.79 & 8.87 & 98.11 \\
11 & 13.55 & 11.14 & 98.02 \\
12 & 12.36 & 16.83 & 98.59 \\
13 & 12.29 & 16.85 & 97.87 \\
14 & 11.44 & 11.52 & 97.00 \\
15 & 11.65 & 7.72 & 98.05 \\
16 & 11.73 & 17.79 & 97.68 \\
\hline
\end{tabular}

the proposed antenna can be easily scaled in order to work at low frequencies, without degrading its performances.

In Figures 7 and 8 we show, respectively, the return loss and the Gain, plotted with respect to the normalized frequency, of the antenna designed with SED within S, C, $\mathrm{X}$, and $\mathrm{Ku}$ bands and of the same antenna scaled at the center frequency of $2 \mathrm{GHz}$. The simulations, performed with NEC-2, show a very similar behavior in the whole operating bandwidth, confirming that the proposed antenna can be easily scaled to work at any lower frequency.

Following [29, 30], a wire LPDA with the same bandwidth of our broadband antenna scaled at $2 \mathrm{GHz}$ (Figures 7 and 8) will require at least 20 elements to get an average gain of only $8.5 \mathrm{~dB}$, with the $\log$ period $\tau$ equal to 0.9 . However, $\tau$ should be kept lower than 0.85 in order to ensure a good behaviour of the LPDA [30], and this constraint limits the gain of a standard LPDA to a value below $8 \mathrm{~dB}$. This comparison shows that the proposed antenna allows significantly better performances with respect to standard LPDAs, with a little bit more complicated structure, but without requiring the typical twisted-cable feeding network of the LPDAs.

\section{Conclusion}

A new design technique for a wideband wire antenna has been presented. It is based on the Structure-based Evolutionary Design (SED), which exploits the concept of the 
Evolutionary Programming. Since no a priori structure is assumed, a suitable fitness function allows to reach significant electrical performances with a simple geometry. Extension to multiobjective fitness is under consideration, but the results reported here show that its use is not required, except perhaps for very complicate requirements.

Inclusion of the ohmic losses and of a suitable robustness test leads to a small antenna size, while preventing from super directive solutions. The proposed approach can therefore be effectively employed also for different sets of requirements.

\section{Conflict of Interests}

The authors declare that there is no conflict of interests.

\section{References}

[1] S. Silver, Microwave Antenna Theory and Design, McGraw-Hill, 1949.

[2] R. S. Elliott, Antenna Theory and Design, Prentice-Hall, New York, NY, USA, 1981.

[3] R. E. Collin, Antennas and Radiowave Propagation, McGrawHill, 1986.

[4] H. J. Orchard, R. S. Elliott, and G. J. Stern, "Optimising the synthesis of shaped beam antenna patterns," IEE Proceedings $H$, vol. 132, no. 1, pp. 63-68, 1985.

[5] G. A. Casula and G. Montisci, "Design of dielectric-covered planar arrays of longitudinal slots," IEEE Antennas and Wireless Propagation Letters, vol. 8, pp. 752-755, 2009.

[6] O. M. Bucci, G. D’Elia, G. Mazzarella, and G. Panariello, "Antenna pattern synthesis. A new general approach," Proceedings of the IEEE, vol. 82, no. 3, pp. 358-371, 1994.

[7] G. Montisci, "Design of circularly polarized waveguide slot linear arrays," IEEE Transactions on Antennas and Propagation, vol. 54, no. 10, pp. 3025-3029, 2006.

[8] G. A. Casula, G. Mazzarella, and G. Montisci, "Effect of the feeding t-junctions in the performance of planar waveguide slot arrays," IEEE Antennas and Wireless Propagation Letters, vol. 11, pp. 953-956, 2012.

[9] G. A. Casula, G. Mazzarella, and G. Montisci, "A truncated waveguide fed by a microstrip as radiating element for high performance automotive anti-collision radars," International Journal of Antennas and Propagation, vol. 2012, Article ID 983281, 9 pages, 2012.

[10] S. Costanzo, G. A. Casula, A. Borgia et al., "Synthesis of slot arrays on integrated waveguides," IEEE Antennas and Wireless Propagation Letters, vol. 9, pp. 962-965, 2010.

[11] Z. Jin, G. Montisci, G. A. Casula, H. Yang, and J. Lu, "Efficient evaluation of the external mutual coupling in dielectric-covered waveguide slot arrays," International Journal of Antennas and Propagation, vol. 2012, Article ID 491242, 7 pages, 2012.

[12] R. Carrel, “The design of log-periodic dipole antennas," IRE International Convention Record, vol. 9, pp. 61-75, 1961.

[13] R. Mishra, R. Ghatak, and D. Poddar, "Design formula for Sierpinski Gasket pre-fractal planar-monopole antennas," IEEE Antennas and Propagation Magazine, vol. 50, no. 3, pp. 104-107, 2008.

[14] S. Congiu and G. Mazzarella, "A tri-band printed antenna based on a Sierpinski gasket," Journal of Electromagnetic Waves and Applications, vol. 21, no. 15, pp. 2187-2200, 2007.
[15] G. K. Mahanti, A. Chakrabarty, and S. Das, "Phase-only and amplitude-phase synthesis of dual-pattern linear antenna arrays using floating-point genetic algorithms," Progress in Electromagnetics Research, vol. 68, pp. 247-259, 2007.

[16] W. B. Wang, Q. Y. Feng, and D. Liu, "Application of chaotic particle swarm optimization algorithm to pattern synthesis of antenna arrays," Progress in Electromagnetics Research, vol. 115, pp. 173-189, 2011.

[17] S. A. Hosseini and Z. Atlasbaf, "Optimization of side lobe level and fixing quasi-nulls in both of the sum and difference patterns by using Continuous Ant Colony Optimization (ACO) method," Progress in Electromagnetics Research, vol. 79, pp. 321-337, 2008.

[18] K.-C. Lee, "Frequency-domain analyses of nonlinearly loaded antenna arrays using simulated annealing algorithms," Progress in Electromagnetics Research, vol. 53, pp. 271-281, 2005.

[19] B. Mhamdi, K. Grayaa, and T. Aguili, "Hybrid of particle swarm optimization, simulated annealing and tabu search for the reconstruction of two-dimensional targets from laboratorycontrolled data," Progress In Electromagnetics Research B, no. 28, pp. 1-18, 2011.

[20] S. Baskar, A. Alphones, P. N. Suganthan, and J. J. Liang, "Design of Yagi-Uda antennas using comprehensive learning particle swarm optimization," IEE Proceedings, vol. 152, pp. 340-346, 2005.

[21] D. S. Linden and E. F. Altshuler, "Automating wire antenna design using genetic algorithms," Microwave Journal, vol. 39, no. 3, pp. 74-86, 1996.

[22] D. S. Linden and E. E. Altshuler, "Wire-antenna designs using genetic algorithms," IEEE Antennas and Propagation Magazine, vol. 39, no. 2, pp. 33-43, 1997.

[23] E. A. Jones and W. T. Joines, "Design of yagi-uda antennas using genetic algorithms," IEEE Transactions on Antennas and Propagation, vol. 45, no. 9, pp. 1386-1392, 1997.

[24] G. A. Casula, G. Mazzarella, and N. Sirena, "Evolutionary design of wide-band parasitic dipole arrays," IEEE Transactions on Antennas and Propagation, vol. 59, pp. 4094-4102, 2011.

[25] J. R. Koza, M. A. Keane, M. J. Streeter, W. Mydlowec, J. Yu, and G. Lanza, Genetic Programming IV: Routine HumanCompetitive Machine Intelligence, Springer, 2003.

[26] J. D. Lohn, G. S. Hornby, and D. S. Linden, "An evolved antenna for deployment on NASA's space technology 5 mission," in Genetic Programming Theory and Practice II, Springer, 2005.

[27] L. Deias, G. Mazzarella, G. Montisci, and G. A. Casula, "Synthesis of artificial magnetic conductors using structurebased evolutionary design," submitted to International Journal of Antennas and Propagation.

[28] W. L. Stutzman and G. A. Thiele, Antenna Theory and Design, John Wiley, New York, NY, USA, 2nd edition, 1997.

[29] D. E. Isbell, "Log periodic dipole antennas," IRE Transactions on Antennas and Propagation, vol. 8, pp. 260-267, 1960.

[30] R. Carrel, "The design of log-periodic dipole antennas," IRE International Convention Record, pp. 61-75, 1961.

[31] G. J. Burke and A. J. Poggio, "Numerical Electromagnetics Code-Method of Moments," Tech. Rep. UCID-18834, Lawrence Livermore National Laboratory, 1981.

[32] G. J. Burke and A. J. Poggio, "Computer Analysis of the Twin-Whip," Tech. Rep. UCRL-52080, Lawrence Livermore Laboratory, Calif, USA, 1976.

[33] G. J. Burke and A. J. Poggio, "Computer Analysis of the Bottom-Fed FM Antenna," Tech. Rep. UCRL-52109, Lawrence Livermore Laboratory, Calif, USA, 1976. 
[34] D. S. Linden and E. E. Altshuler, "Wire-antenna designs using genetic algorithms," IEEE Antennas and Propagation Magazine, vol. 39, no. 2, pp. 33-43, 1997.

[35] http://www.ansys.com/Products/Simulation+Technology/ Electromagnetics/High-Performance+Electronic+Design/ ANSYS+HFSS.

[36] S. R. Rengarajan, M. S. Zawadzki, and R. E. Hodges, "Waveguide-slot array antenna designs for low-average-sidelobe specifications," IEEE Antennas and Propagation Magazine, vol. 52, no. 6, pp. 89-98, 2010.

[37] B. C. Wadell, Transmission Line Design Handbook, Artech House, 1991.

[38] G. A. Casula, G. Mazzarella, and N. Sirena, "On the effect of the finite metal conductivity in the design of wire antennas using evolutionary design," in Proceedings of the Antennas and Propagation Society International Symposium, 2011. 

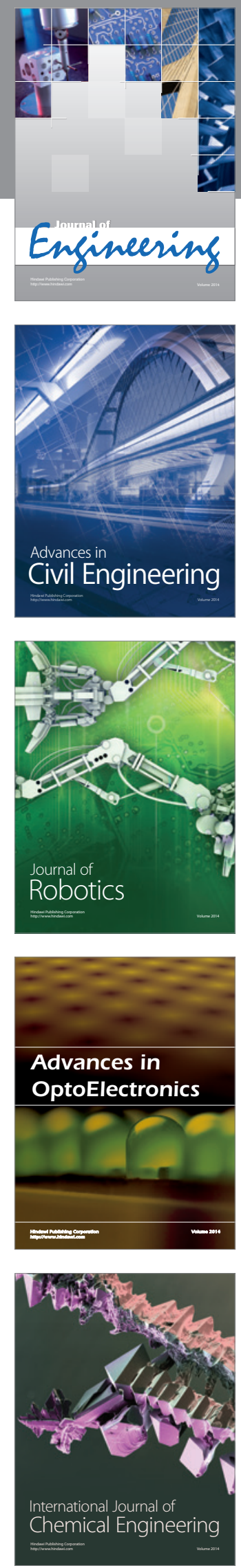

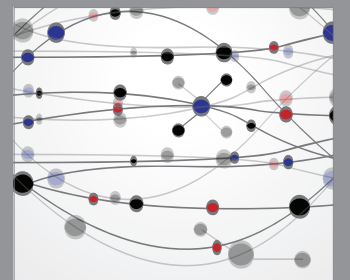

The Scientific World Journal
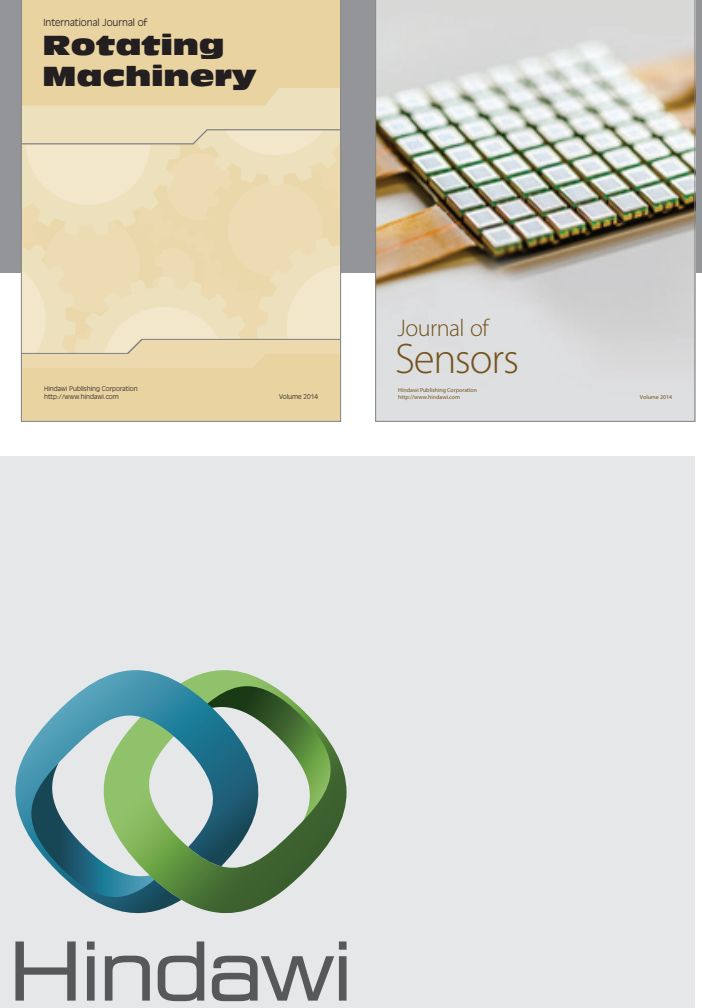

Submit your manuscripts at http://www.hindawi.com
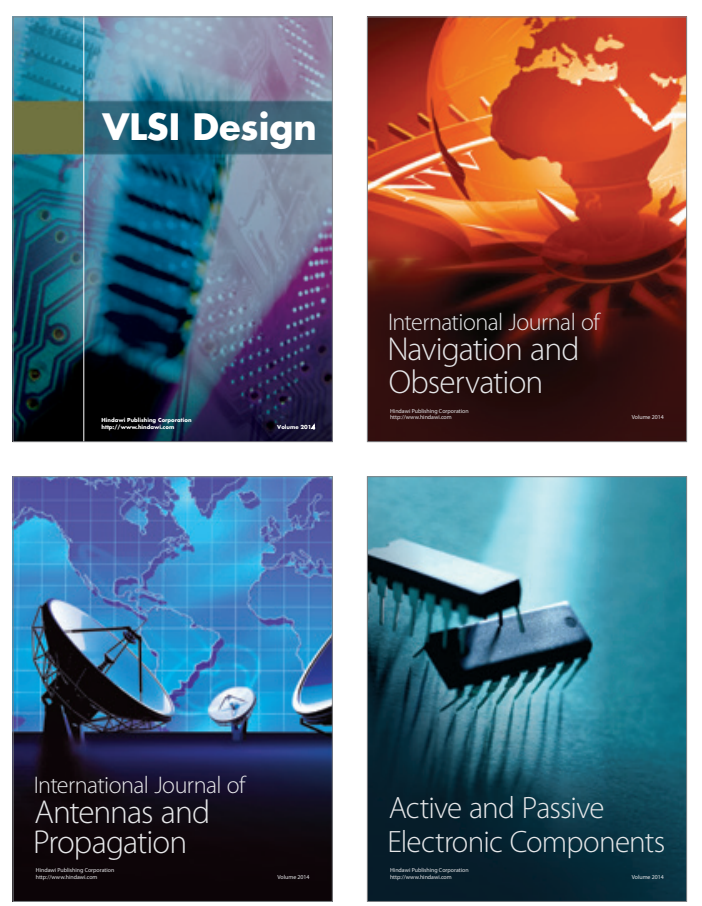
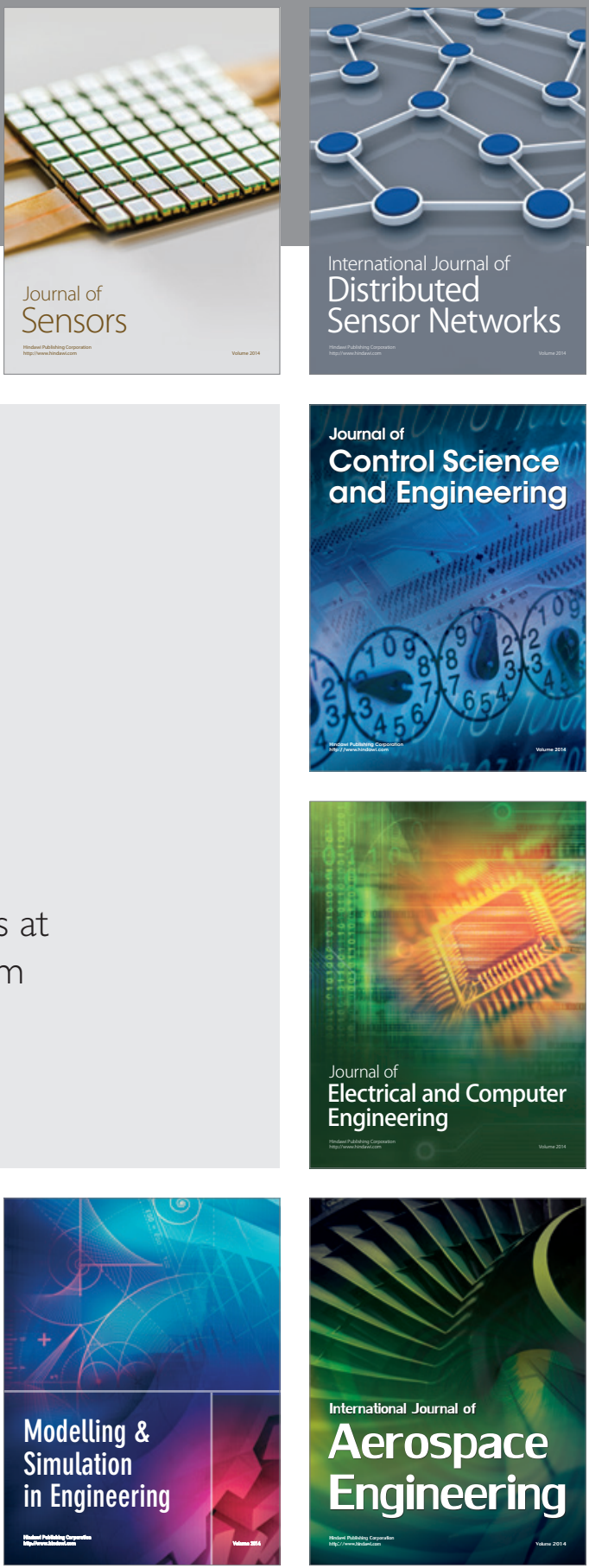

Journal of

Control Science

and Engineering
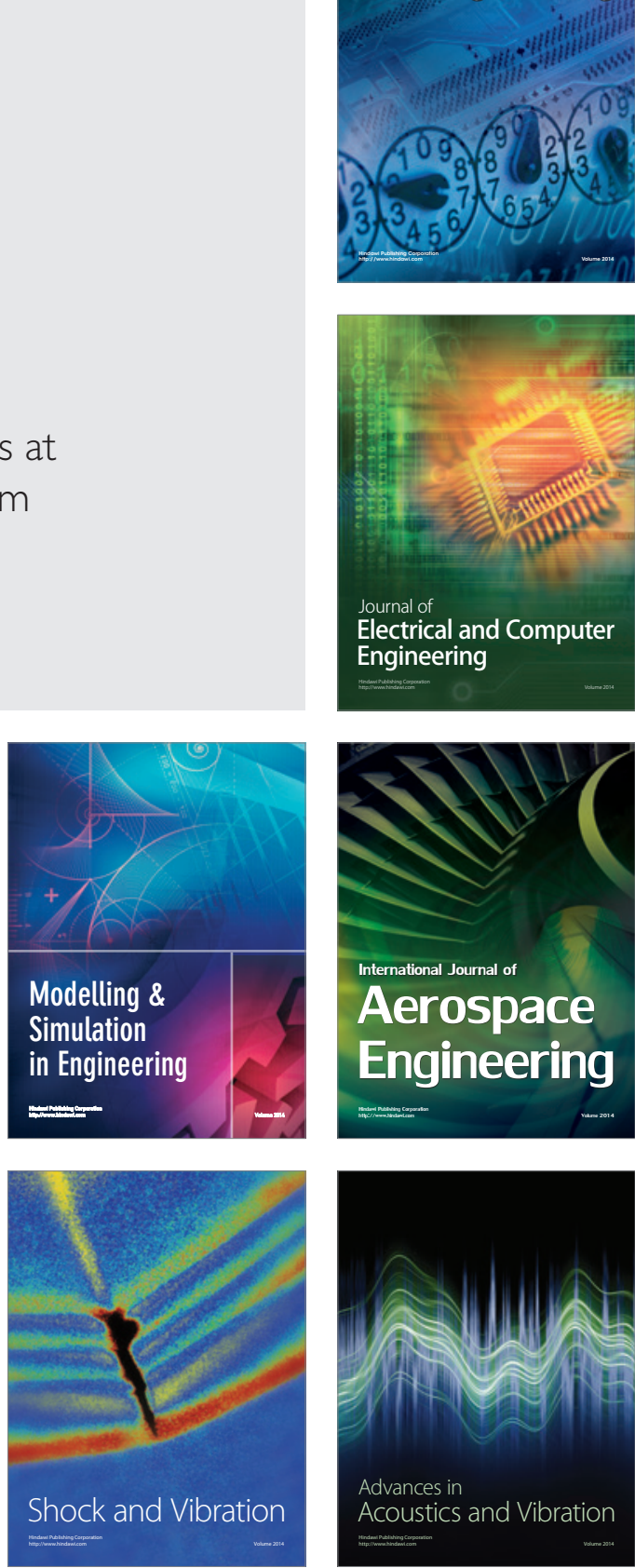